W. Montero P. et al. DR2013140

This supplemental information file for "Neotectonic faulting and forearc sliver motion along the Atirro-Río Sucio fault system, Costa Rica, Central America” by Montero et al. contains descriptions of secondary faults of Atirro-Río Sucio fault system.

\title{
Omega fault
}

The Omega fault is an Atirro fault splay that branches westward off of the main trace at the Rincón de la Esperanza pressure ridge (Fig. 3 in manuscript). This fault traverses a series of linear valleys exhibiting abrupt scarps, dextral stream offsets, and drainage adaptations (Montero, 2003). The fault loses expression to the west-northwest where it truncates against the Pejibaye pull-apart basin. Where the Pejibaye River crosses the Omega ridge, it enters a narrow canyon (site 8, Fig. 7 in manuscript) that we interpret as an antecedent valley. Uplift here is thought to reflect a transpressive left step between the Atirro and Omega faults (Montero, 2003) (Fig. 3 in manuscript). In the past, the Omega fault may have continued to the northwest along either of the two arms of the Tucurrique fault that bound the Pejibaye pull-apart. If the Omega fault continued along the northern arm, it is now offset $0.5 \mathrm{~km}$ by dextral motion on the basin margin. If it continued along the Tucurrique fault's southern arm, the old trace is now covered by basin alluvium.

\section{Chiz and Campano faults}

The linear Chiz fault is one of several sub-parallel fault traces near the basin boundary (Fig. 3 in manuscript). The fault's southern end near the town of Tucurrique marks the contact between Pliocene volcanic rocks exposed in hills to the east, and Quaternary volcanic terraces to the west. The prominent scarp of this normal-slip fault reaches a maximum height of $500 \mathrm{~m}$ along the Alto Florencia crestal line (Figs. 7 and 9 in manuscript). Windows of Tertiary sedimentary rocks appear along the scarp surrounded by Quaternary lava flows (Fernández, 
1987; Mora et al., 1990). The northeastern flank of the Alto Florencia has a gentler slope than the southwestern flank, causing an asymmetric drainage network in which streams are longer toward the northeast. Based on this asymmetry, the eastern block is tilted down to the northeast. The Campano fault (manuscript Figs. 3 and 7), with down-to-the-east normal displacement, is inferred to mark the southwestern boundary of the Irazú-Turrialba pull-apart basin. It has a prominent triangular-faceted scarp, up to $300 \mathrm{~m}$ in height. South of the town of Tucurrique, the fault is coincident with a right deflection of the Vueltas river and with a beheahed stream afluent of the Pisirí creek (BC in manuscript Fig. 7), suggesting combined dextral and normal displacements.

\section{Faults of the Irazú-Turrialba volcanic field}

The Ariete-Río Guácimo fault (ARF in Fig. DR1) on Turrialba volcano is characterized by well defined $25 \mathrm{~m}$ scarps. South and west of Irazú volcano, several rectilinear to curvilinear fault traces stand out. The Irazú fault (Fig. DR1) displays a clear northwest-facing scarp with downthrown western side. A more curvilinear sub-parallel fault to the southeast also displays northwest-facing scarps. On the western side of Irazú volcano two curvilinear faults show faceted scarps and linear valleys (Fig. 2 in manuscript). A north-northeast alignment of monogenetic volcanoes including the Irazú active summit crater suggests an E-W to WNW extension within the Irazú volcanic field. This extension is concordant with the tensional stresses that open the Irazú-Turrialba pull-apart (Fig. DR1).

\section{The splays of the Río Sucio fault}

The easternmost Río Sucio fault splay (Figs. 10 and 11 in manuscript) is expressed by linear valleys and southwest-facing faceted $200-400 \mathrm{~m}$ scarps. A transtensive relay near the southeast 
end of the easternmost splay suggests a dextral component of slip (Figs. 10 and 11 in manuscript; Montero, 2003). Further east the easternmost fault splay is interrupted by the Finca Liebres volcanic depression. The central Río Sucio fault branch is also characterized by linear valleys and southwest-facing scarps. At the north end of the western Río Sucio fault branch, a small basin in a right stepping bend (Fig. 10 in manuscript) implies a releasing geometry and dextral slip along the fault.

\section{The Blanquito fault}

On the northern slopes of the Irazú-Turrialba volcanoes, Montero and Alvarado (1995) described some NNW-trending neotectonic faults. The most well expressed is the Blanquito fault (Figs. 2 and 10 in manuscript). The southern termination cuts the Finca Liebres volcanic depression. The fault is expressed by west facing scarps, linear valleys and adapted and displaced rivers. The northern end of the fault is located south of the Guápiles thrust fault (Fig. 10 in manuscript).

\section{References}

Dziewonski, A.M., Ekström, G., Woodhouse, J.H., and Zwart, G., 1989, Centroid-moment tensor solutions for January-March, 1988: Physics of the Earth and Planetary Interiors, v. 54, p. 22-32, doi:10.1016/0031-9201(89)90183-0.

Dziewonski, A.M., Ekström, G., and Salganik, M.P., 1994, Centroid-moment tensor solutions for July-September 1993: Physics of the Earth and Planetary Interiors, v. 83, p. 165-174, doi:10.1016/0031-9201(94)90087-6.

Fernández, J.A., 1987, Geología de la Hoja Topográfica Tucurrique [Licenciatura thesis]: San Pedro, Universidad de Costa Rica, 205 p. 
Fernández, M., and Pacheco, J., 1998, Sismotectónica de la región central de Costa Rica: Revista Geológica América Central, v. 21, p. 5-23.

Mora, R., Cerdas, A., Molina, F., and Vega, E., 1990, Caracterización geológica del deslizamiento Chiz (Turrialba, Provincia de Cartago, Costa Rica): Amenaza y prevención: Revista Geológica América Central, v. 11, p. 59-68. 


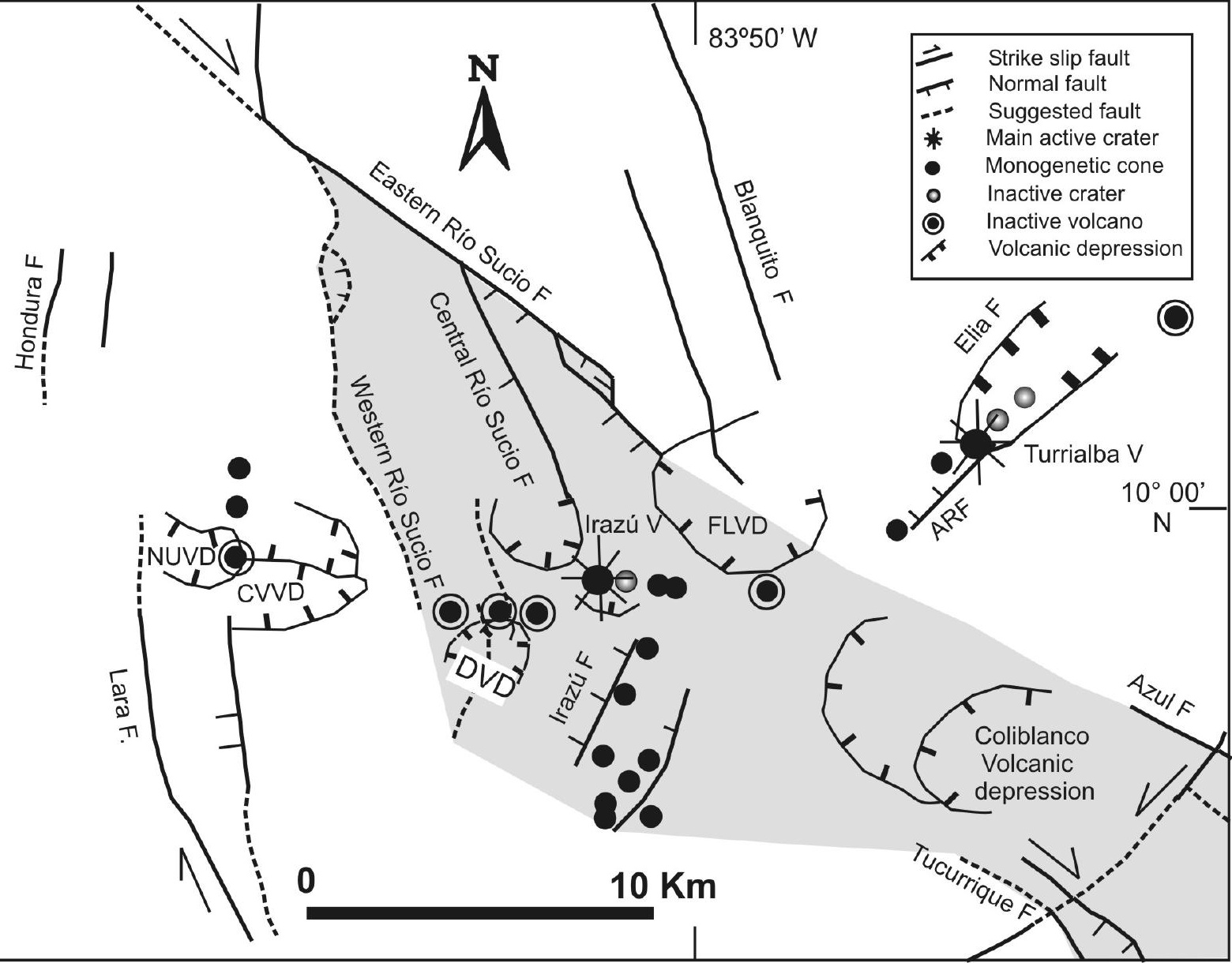




\begin{tabular}{|c|c|c|c|c|c|c|}
\hline Fault & Map & Figure & Offset & & & Piercing points and \\
\hline name & symbol & number & feature & $\begin{array}{c}\text { minimum } \\
(\mathrm{km})\end{array}$ & $\begin{array}{c}\text { maximum } \\
(\mathrm{km})\end{array}$ & \\
\hline
\end{tabular}

Oriente

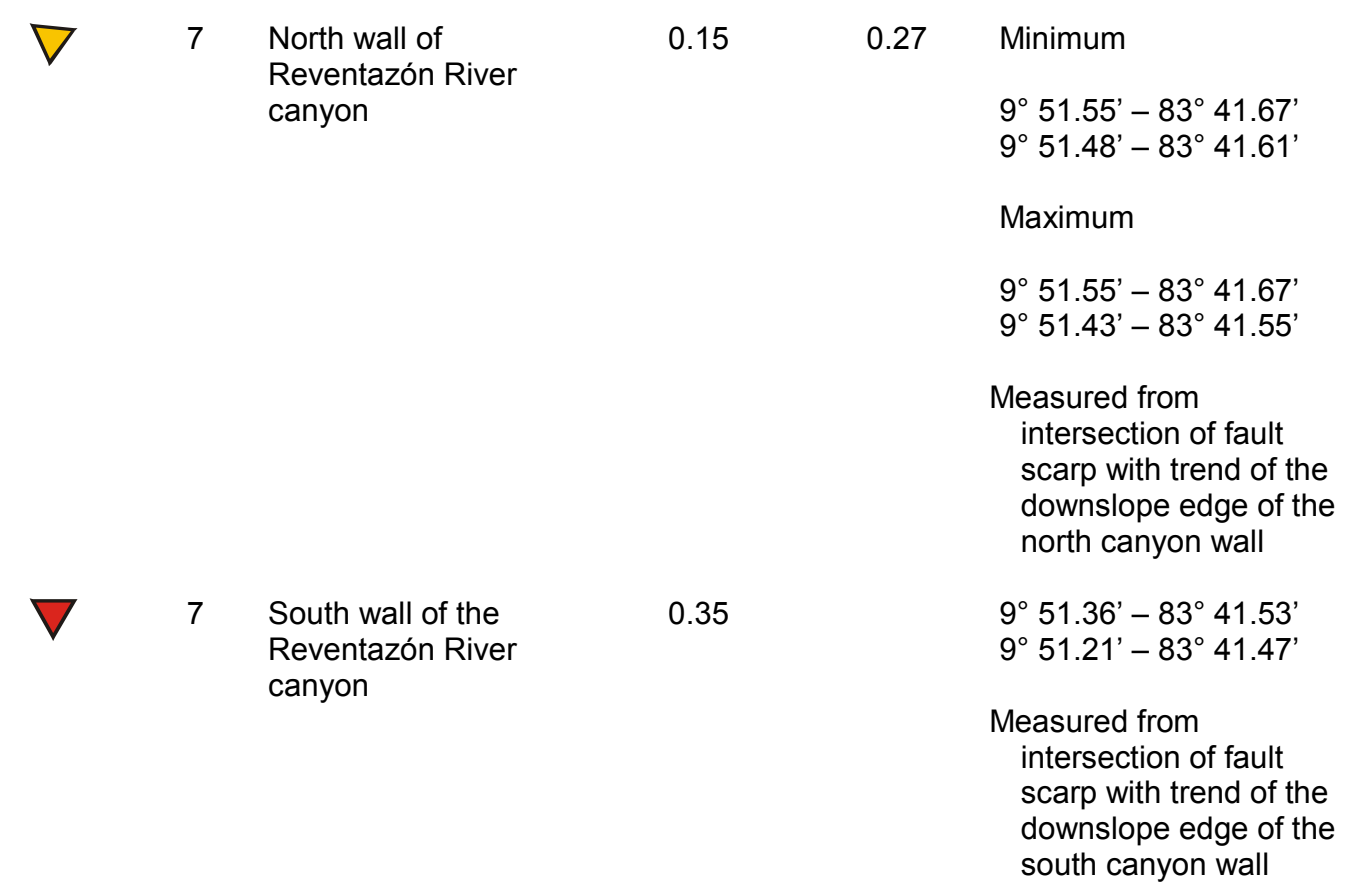

$\underline{\text { Tucurrique }}$

$7 \quad$ Downslope edge of lower volcanic terrace

7

Downslope edge of lower volcanic terrace

$\bigcirc$
7 Contact between Pliocene volcanic basement and lower volcanic terrace $9^{\circ} 51.74^{\prime}-83^{\circ} 43.30^{\prime}$

$9^{\circ} 51.62^{\prime}-83^{\circ} 43.23^{\prime}$

Measured parallel to trend of the downslope edge of the terraces

$0.55 \quad 9^{\circ} 51.74^{\prime}-83^{\circ} 43.30^{\prime}$

$9^{\circ} 51.49^{\prime}-83^{\circ} 43.15^{\prime}$

Measured away from the fault intersection and perpendicular to fault trace

$\sim 0.30$

$$
\begin{aligned}
& 9^{\circ} 51.07^{\prime}-83^{\circ} 43.04^{\prime} \\
& 9^{\circ} 50.91^{\prime}-83^{\circ} 43.01^{\prime} \\
& \text { Measured from } \\
& \text { intersection of fault }
\end{aligned}
$$


scarp with trace of contacts

$7 \quad \begin{aligned} & \text { Contact between } \\ & \text { Pliocene volcanic } \\ & \text { basement and lower } \\ & \text { volcanic terrace }\end{aligned}$

volcanic terrace $\sim 0.80$

$9^{\circ} 51.07^{\prime}-83^{\circ} 43.04^{\prime}$

$9^{\circ} 50.71^{\prime}-83^{\circ} 42.43^{\prime}$

Measured from intersection of fault scarp with trace of contacts.

Displacement accumulated between two fault traces

Turrialba

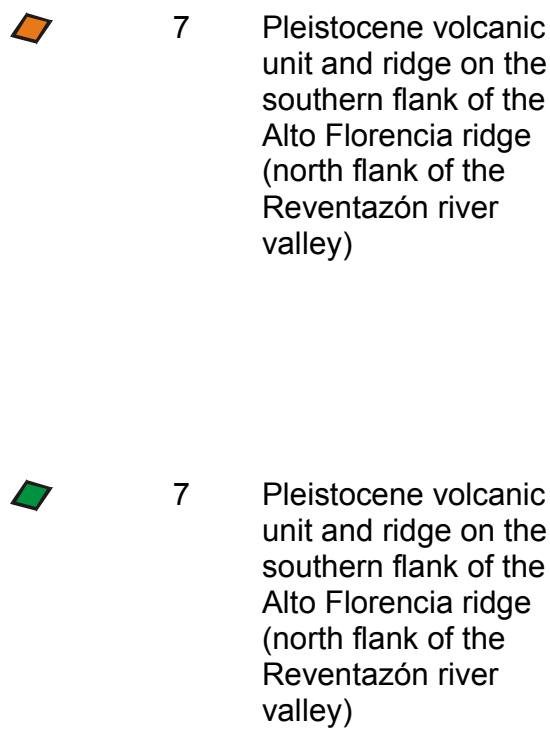

$\sim 0.95$

$9^{\circ} 51.96^{\prime}-83^{\circ} 40.64^{\prime}$

$9^{\circ} 51.39^{\prime}-83^{\circ} 40.64^{\prime}$

Measured from trend of contact to fault trace $\sim 1.20 \quad 9^{\circ} 51.96^{\prime}-83^{\circ} 40.64^{\prime}$

$9^{\circ} 51.39^{\prime}-83^{\circ} 40.33^{\prime}$

Measured from trend of contact to eastern fault trace

* Estimates of fault displacement where topographic gradients are steep along the Reventazón River are considered accurate to approximately $\pm 10 \mathrm{~m}$. In all other areas topographic gradients are less steep and colluvial cover is more abundant, therefore the estimates of displacement are considered accurate to approximately $\pm 100 \mathrm{~m}$. 
TABLE DR2: FOCAL MECHANISMS ALONG THE ATIRRO-RÍO SUCIO FAULT SYSTEM, COSTA RICA, M $\geq 4.0$

\begin{tabular}{|c|c|c|c|c|c|c|c|}
\hline \multirow{2}{*}{$\begin{array}{l}\text { Event } \\
\text { no.* }\end{array}$} & \multirow{2}{*}{$\begin{array}{l}\text { Event timing }^{\dagger} \\
\text { DDMMYYHrMi }\end{array}$} & \multicolumn{3}{|c|}{ Location $^{\S}$} & \multirow[t]{2}{*}{ Magnitude } & \multicolumn{2}{|c|}{ Mechanism $^{* *}$} \\
\hline & & $\begin{array}{l}\text { Latitude } \\
\qquad\left({ }^{\circ} \mathrm{N}\right)\end{array}$ & $\begin{array}{l}\text { Longitude } \\
\qquad\left({ }^{\circ} \mathrm{W}\right)\end{array}$ & $\begin{array}{c}\text { Depth } \\
(\mathrm{km})\end{array}$ & & $\begin{array}{c}\text { Nodal Plane } \\
\text { (Az/Dp/Rk) }\end{array}$ & $\begin{array}{l}\text { P Axis } \\
\text { (Az/Ln) }\end{array}$ \\
\hline $1^{1}$ & 1911871602 & 09.733 & 83.584 & 19 & $4.5 \mathrm{~m}_{\mathrm{b}} 4.3 \mathrm{M}_{\mathrm{s}}$ & $309 / 33 / 117$ & $200 / 14$ \\
\hline $2^{2}$ & 3101882331 & 09.870 & 83.610 & (15) & $4.8 \mathrm{~m}_{\mathrm{b}} 4.4 \mathrm{M}_{\mathrm{s}}$ & 239/90/000 & $012 / 00$ \\
\hline $3^{3}$ & 2108901915 & 10.073 & 83.964 & 14 & $4.4 \mathrm{M}_{\mathrm{d}} 4.4 \mathrm{~m}_{\mathrm{b}}$ & 145/80/090 & $235 / 35$ \\
\hline $4^{4}$ & 2504910755 & 09.606 & 83.568 & 12 & $4.7 \mathrm{M}_{\mathrm{d}}$ & $273 / 33 / 075$ & $194 / 77$ \\
\hline $5^{4}$ & 2504911432 & 09.888 & 83.503 & 14 & $4.7 \mathrm{M}_{\mathrm{d}}$ & $112 / 80 / 057$ & $176 / 63$ \\
\hline $6^{4}$ & 2704912259 & 09.897 & 83.396 & 14 & $4.0 \mathrm{M}_{\mathrm{d}}$ & 103/60/052 & $167 / 82$ \\
\hline $7^{4}$ & 2904911249 & 09.761 & 83.910 & 23 & $4.7 \mathrm{M}_{\mathrm{d}}$ & $033 / 74 /-021$ & $256 / 64$ \\
\hline $8^{5}$ & 1405911953 & 09.866 & 83.525 & 21 & $4.4 \mathrm{~m}_{\mathrm{b}} 4.2 \mathrm{M}_{\mathrm{d}}$ & $171 / 74 /-177$ & $033 / 13$ \\
\hline $9^{5}$ & 1705910122 & 09.838 & 83.397 & 18 & $4.0 \mathrm{M}_{\mathrm{d}}$ & $220 / 52 / 043$ & $161 / 03$ \\
\hline $10^{6}$ & 0506911134 & 09.983 & 83.775 & 06 & $4.2 \mathrm{M}_{\mathrm{d}}$ & $177 / 64 / 136$ & $240 / 13$ \\
\hline $11^{6}$ & 0506911140 & 10.002 & 83.766 & 05 & $4.0 \mathrm{M}_{\mathrm{d}}$ & $180 / 40 /-167$ & $027 / 40$ \\
\hline $12^{6}$ & 2006910811 & 09.958 & 83.854 & 05 & $4.3 \mathrm{M}_{\mathrm{d}}$ & 050/70/-044 & $000 / 44$ \\
\hline $13^{3}$ & 0212922103 & 09.975 & 84.000 & 18 & $4.0 \mathrm{M}_{\mathrm{d}}$ & $250 / 85 /-085$ & $166 / 50$ \\
\hline $14^{1}$ & 0807932319 & 09.748 & 83.687 & 14 & $4.7 \mathrm{~m}_{\mathrm{b}} 4.6 \mathrm{M}_{\mathrm{s}}$ & 068/61/-042 & $032 / 50$ \\
\hline $15^{3}$ & 0907930200 & 09.755 & 83.688 & 16 & $4.3 \mathrm{M}_{\mathrm{d}}$ & 253/83/-029 & $206 / 26$ \\
\hline $16^{\prime}$ & 1007932041 & 09.756 & 83.677 & 14 & $5.3 \mathrm{~m}_{\mathrm{b}} 5.6 \mathrm{M}_{\mathrm{s}} 5.8 \mathrm{M}_{\mathrm{w}}$ & $168 / 90 /-180$ & $033 / 00$ \\
\hline $17^{1}$ & 1307931510 & 09.724 & 83.657 & 13 & $4.6 \mathrm{~m}_{\mathrm{b}} 4.8 \mathrm{M}_{\mathrm{s}}$ & 260/73/-058 & $208 / 55$ \\
\hline $18^{8}$ & 1606970905 & 09.963 & 83.832 & 03 & $4.0 \mathrm{M}_{\mathrm{d}}$ & 221/48/039 & $164 / 08$ \\
\hline
\end{tabular}

* Focal mechanism numbers shown in figure 12. The superscripts refer to the data sources as follows: ${ }^{1}$ Montero (2001); ${ }^{2}$ Dziewonski et al. (1989); ${ }^{3}$ Fernández and Pacheco (1998); ${ }^{4}$ Barquero and Rojas (1994); ${ }^{5}$ Fan et al. (1993); ${ }^{6}$ Barquero et al. (1995); ${ }^{7}$ Dziewonski et al. (1994); and ${ }^{8}$ Fernández et al. (1998).

†Timing given as follows: $\mathrm{DD}=$ day; $\mathrm{MM}=$ month; $\mathrm{YY}=$ year; $\mathrm{Hr}=$ hour and $\mathrm{Mi}=$ minute.

§The location for Event 16 comes from the Costa Rica Regional Seismic Network (RSN). Depth in paretheses indicates centroid tensor moment (CMT) depth.

\#Magnitude is given using the following formats: $m_{b}=$ body wave magnitude; $M_{s}=$ surface wave magnitude and $M_{d}=$ duration magnitude

${ }^{* *}$ Mechanism columns use the following abbreviations: $\mathrm{Az}=$ azimuth; $\mathrm{Dp}=\mathrm{dip}$; $\mathrm{Rk}=$ rake of slip vector measured from strike such that nodal plane dips to the right (i.e., using the right hand rule) and Ln is the inclination of the $P$ axis. 
TABLE DR3. MESOSCALE FAULT POPULATION DATA

\begin{tabular}{|c|c|c|c|c|c|c|c|c|}
\hline \multicolumn{2}{|c|}{ Fault } & \multicolumn{2}{|c|}{ Striae } & \multirow{2}{*}{$\begin{array}{c}\text { Slip } \\
\text { Sense }\end{array}$} & \multicolumn{2}{|c|}{ T-axis } & \multicolumn{2}{|c|}{ P-axis } \\
\hline Strike & Dip & Trend & Plunge & & Trend & Plunge & Trend & Plunge \\
\hline \multicolumn{9}{|c|}{ FP 81 - Tajo Oriente, Río Pejibaye } \\
\hline 265 & 74 & 278 & 38 & NL & 321 & 14 & 219 & 39 \\
\hline 318 & 82 & 321 & 20 & TR & 273 & 20 & 006 & 08 \\
\hline 282 & 86 & 282 & 06 & NL & 327 & 01 & 237 & 07 \\
\hline 216 & 87 & 025 & 75 & NR & 320 & 40 & 111 & 46 \\
\hline 123 & 81 & 296 & 37 & NR & 249 & 18 & 351 & 32 \\
\hline 102 & 70 & 107 & 13 & NL & 329 & 05 & 061 & 24 \\
\hline 333 & 80 & 335 & 10 & TR & 289 & 14 & 019 & 00 \\
\hline 055 & 84 & 234 & 05 & TL & 279 & 08 & 010 & 01 \\
\hline 054 & 81 & 221 & 54 & TL & 290 & 43 & 171 & 27 \\
\hline 340 & 70 & 154 & 15 & NR & 293 & 03 & 201 & 25 \\
\hline 311 & 73 & 314 & 10 & TR & 268 & 19 & 177 & 05 \\
\hline 318 & 64 & 323 & 11 & TR & 278 & 26 & 183 & 10 \\
\hline 062 & 64 & 067 & 11 & NL & 287 & 10 & 022 & 26 \\
\hline 075 & 48 & 089 & 15 & NL & 301 & 17 & 046 & 41 \\
\hline 030 & 66 & 162 & 59 & TL & 267 & 63 & 135 & 19 \\
\hline 038 & 68 & 190 & 49 & TL & 265 & 53 & 153 & 16 \\
\hline 072 & 83 & 089 & 67 & TR & 005 & 48 & 143 & 34 \\
\hline 076 & 78 & 112 & 70 & TR & 006 & 54 & 153 & 31 \\
\hline \multicolumn{9}{|c|}{ FP 82 - Tajo Esperanza, Río Atirro } \\
\hline 295 & 58 & 306 & 18 & TR & 259 & 36 & 163 & 09 \\
\hline 303 & 80 & 305 & 13 & TR & 259 & 16 & 349 & 02 \\
\hline 338 & 82 & 156 & 14 & NR & 111 & 04 & 203 & 16 \\
\hline 067 & 57 & 243 & 07 & TL & 285 & 28 & 024 & 17 \\
\hline 045 & 65 & 050 & 11 & NL & 270 & 09 & 005 & 26 \\
\hline 336 & 85 & 336 & 05 & TR & 291 & 07 & 021 & 00 \\
\hline 286 & 40 & 075 & 23 & TL & 118 & 55 & 231 & 15 \\
\hline 112 & 80 & 115 & 15 & TR & 068 & 18 & 159 & 03 \\
\hline 025 & 34 & 037 & 08 & NL & 243 & 29 & 005 & 43 \\
\hline 038 & 50 & 048 & 11 & NL & 262 & 19 & 006 & 36 \\
\hline 286 & 82 & 298 & 55 & NL & 349 & 29 & 229 & 43 \\
\hline 054 & 84 & 231 & 30 & TL & 282 & 25 & 184 & 16 \\
\hline 040 & 16 & 140 & 16 & TL & 144 & 61 & 318 & 29 \\
\hline 097 & 85 & 099 & 20 & NL & 145 & 10 & 051 & 18 \\
\hline 242 & 73 & 060 & 07 & TL & 105 & 17 & 197 & 07 \\
\hline 050 & 81 & 053 & 16 & $\mathrm{NL}$ & 097 & 05 & 006 & 18 \\
\hline 042 & 38 & 080 & 26 & NL & 281 & 14 & 037 & 60 \\
\hline 234 & 38 & 254 & 15 & $\mathrm{NL}$ & 100 & 22 & 217 & 48 \\
\hline 282 & 75 & 284 & 08 & TR & 239 & 16 & 147 & 05 \\
\hline 092 & 76 & 266 & 21 & TL & 315 & 25 & 223 & 05 \\
\hline 261 & 74 & 269 & 27 & NL & 312 & 07 & 218 & 31 \\
\hline 065 & 77 & 242 & 12 & TL & 288 & 18 & 019 & 01 \\
\hline 053 & 65 & 057 & 09 & NL & 278 & 11 & 013 & 24 \\
\hline 083 & 40 & 226 & 27 & TL & 272 & 59 & 024 & 13 \\
\hline 070 & 50 & 230 & 23 & TL & 278 & 47 & 019 & 10 \\
\hline 259 & 85 & 078 & 12 & TL & 124 & 12 & 033 & 05 \\
\hline 098 & 80 & 277 & 05 & NR & 053 & 04 & 322 & 11 \\
\hline 085 & 67 & 228 & 55 & TL & 316 & 59 & 195 & 18 \\
\hline 265 & 86 & 079 & 56 & $\mathrm{TL}$ & 144 & 39 & 023 & 32 \\
\hline \multicolumn{9}{|c|}{ FP 83 - Tajo Las Quebradas, Bajo Pacuare } \\
\hline 270 & 40 & 358 & 40 & TR & 348 & 85 & 179 & 05 \\
\hline 186 & 53 & 228 & 41 & TR & 158 & 63 & 253 & 02 \\
\hline 180 & 64 & 185 & 11 & TR & 140 & 26 & 045 & 10 \\
\hline 283 & 57 & 013 & 57 & TR & 193 & 78 & 013 & 12 \\
\hline 164 & 68 & 210 & 61 & TR & 104 & 62 & 239 & 21 \\
\hline 152 & 68 & 193 & 59 & TR & 095 & 60 & 225 & 20 \\
\hline 142 & 87 & 147 & 59 & TR & 080 & 40 & 206 & 35 \\
\hline 170 & 74 & 189 & 49 & TR & 120 & 47 & 232 & 20 \\
\hline 162 & 78 & 174 & 45 & TR & 112 & 40 & 220 & 21 \\
\hline 154 & 58 & 161 & 11 & TR & 117 & 31 & 019 & 14 \\
\hline 226 & 20 & 272 & 15 & $\mathrm{NL}$ & 103 & 29 & 254 & 58 \\
\hline 295 & 75 & 319 & 57 & TR & 239 & 51 & 002 & 24 \\
\hline
\end{tabular}

